\title{
BMJ Open Permanent work disability before and after ischaemic heart disease or stroke event: a nationwide population-based cohort study in Sweden
}

\author{
Jenni Ervasti, ${ }^{1}$ Marianna Virtanen, ${ }^{1,2}$ Tea Lallukka, ${ }^{1,3}$ Emilie Friberg, ${ }^{2}$ \\ Ellenor Mittendorfer-Rutz, ${ }^{2}$ Erik Lundström, ${ }^{4}$ Kristina Alexanderson ${ }^{2}$
}

To cite: Ervasti J, Virtanen M, Lallukka T, et al. Permanent work disability before and after ischaemic heart disease or stroke event: a nationwide population-based cohort study in Sweden. BMJ Open 2017;7:e017910. doi:10.1136/ bmjopen-2017-017910

- Prepublication history and additional material for this paper are available online. To view please visit the journal (http:// dx.doi.org/10.1136/bmjopen2017-017910).

EPICOH 2017, Edinburgh, August 28-31

Received 26 May 2017 Revised 17 August 2017 Accepted 22 August 2017

\section{CrossMark}

${ }^{1}$ Research and Service Centre of Occupational Health, Finnish Institute of Occupational Health, Helsinki, Finland

${ }^{2}$ Division of Insurance Medicine, Department of Clinical

Neuroscience, Karolinska

Institutet, Stockholm, Sweden

${ }^{3}$ Faculty of Medicine, University of Helsinki, Helsinki, Finland

${ }^{4}$ Division of Neurology,

Department of Clinical

Neuroscience, Karolinska

Institutet, Stockholm, Sweden

Correspondence to

Dr Jenni Ervasti;

jenni.ervasti@ttl.fi

\section{ABSTRACT}

Objectives We examined the risk of disability pension before and after ischaemic heart disease (IHD) or stroke event, the burden of stroke compared with IHD and which factors predicted disability pension after either event. Design A population-based cohort study with follow-up 5 years before and after the event. Register data were analysed with general linear modelling with binary and Poisson distributions including interaction tests for event type (IHD/stroke).

Setting and participants All people living in Sweden, aged 25-60 years at the first event year, who had been living in Sweden for 5 years before the event and had no indication of IHD or stroke prior to the index event in 2006-2008 were included, except for cases in which death occurred within 30 days of the event. People with both IHD and stroke were excluded, resulting in 18480 cases of IHD (65\%) and 9750 stroke cases (35\%).

Primary outcome measures Disability pension.

Results Of those going to suffer IHD or stroke event, $25 \%$ were already on disability pension a year before the event. The adjusted $\mathrm{OR}$ for disability pension at first postevent year was 2.64 -fold $(95 \% \mathrm{Cl} 2.25$ to 3.11$)$ for people with stroke compared with IHD. Economic inactivity predicted disability pension regardless of event type $(0 \mathrm{R}=3.40$; $95 \% \mathrm{Cl} 2.85$ to 4.04). Comorbid mental disorder was associated with the greatest risk ( $\mathrm{OR}=3.60 ; 95 \% \mathrm{Cl} 2.69$ to 4.83) after an IHD event. Regarding stroke, medical procedure, a proxy for event severity, was the largest contributor ( $0 \mathrm{R}=2.27,95 \% \mathrm{Cl} 1.43$ to 3.60$)$.

Conclusions While IHD event was more common, stroke involved more permanent work disability. Demographic, socioeconomic and comorbidity-related factors were associated with disability pension both before and after the event. The results help occupational and other healthcare professionals to identify vulnerable groups at risk for permanent labour market exclusion after such an event.

\section{INTRODUCTION}

Worldwide, $11 \%$ of the total disease burden as measured with disability-adjusted life years is attributed to ischaemic heart disease (IHD) and stroke. ${ }^{1}$ Due to improved treatment of both IHD and stroke contributing
Strengths and limitations of this study

- With large population-based cohort data with reliable register-based measures and no loss to follow-up, we provided information about how ischaemic heart disease (IHD) and stroke events were linked with risk of permanent work disability, that is, disability pension.

- Compared with previous studies focusing on IHD, we had a longer follow-up time-5years-both before and after the event.

- We were able to include a large set of predictors of disability pension, including sociodemographic factors, comorbid conditions and medical procedure.

- The results may help when planning preventive measures for permanent work disability after IHD or stroke event.

- As we were only able to include information that was available in administrative registers, we had no data on quality and outcome of postevent care, individuals' health behaviours or workplace psychosocial factors.

to declining mortality, ${ }^{2}$ and because of the pressures of extended working careers, the proportion of working-age people with cardiovascular disease is likely to increase. While $53 \%-73 \%$ of people suffering a cardiovascular event return to work, ${ }^{3-6}$ significantly higher proportion leaves working life permanently during the years following a cardiovascular event than among people without such diagnosis. ${ }^{7}$ In order to help people with this disease to continue working, it is important to study the risk factors leading to permanent work disability (ie, disability pension) after a cardiovascular event.

Disease severity, comorbidity, female sex, higher age and lower socioeconomic status have been found to predict disability pension after an IHD event. ${ }^{7-12}$ However, we found no previous research that specifically examined the predictors of disability pension after a 
stroke event. Research on stroke has focused on return to work, which has been associated with a less serious disability, younger age, higher socioeconomic position and less cardiovascular risk factors. ${ }^{4-6}$ While IHD and stroke share several common risk factors, some discrepancies also point to differential pattern of predictors. ${ }^{13}$ Previous studies have not examined whether differences exist between the predictors of disability pension after IHD and stroke events.

Our aim was to (A) determine the proportion and characteristics of people who suffered an IHD or stroke event at working age who were already on disability pension prior to the event; and (B) examine the medical (comorbidity, event severity) and non-medical (demographic and socioeconomic) predictors of disability pension in the first postevent year, including examining difference in IHD and stroke cases.

From a labour force policy perspective, it is important to determine whether the predictors of disability pension shortly after the event are different from those that predict disability pension in the longer run. Thus, as a sensitivity analysis, we studied the medical and non-medical predictors of disability pension in the fifth postevent year and whether there were differences between IHD and stroke cases.

\section{METHODS}

\section{Study design}

The population-based longitudinal cohort study was conducted based on register data obtained from three Swedish authorities and linked using the personal identity number assigned to all residents in Sweden. The following registers were used:

1. Statistics Sweden: LISA (Longitudinal Integration Database for Health Insurance and Labour Market Studies) on sex, age, education, family situation, place of birth, type of living area and labour market activity

2. National Board of Health and Welfare: diagnosis-specific data on hospitalisations and specialised outpatient care (coded according to the International Classification of Diseases (ICD-10) ${ }^{14}$ ); medical procedures; cancer register; date of death

3. National Social Insurance Agency: annual sickness absence data (pre-event) and disability pension data.

Our study cohort consisted of all people living in Sweden, who at the event year were aged 25-60 years, had been living in Sweden for 5 years before the event, and had no indication of cardiovascular events in the registers between 2001 and the event year. First event dates in 2006, 2007 and 2008 were included, except for cases in which death occurred within 30 days of the event. This resulted in a sample of 28374 cases. The data on cumulative disability pension were gathered for 5 years prior to the event date, and 5 years after the event. People with both IHD and stroke were excluded $(\mathrm{n}=144)$, resulting in 18480 cases of IHD and 9750 stroke cases.
In prospective analyses on the predictors of disability pension in the first and fifth postevent years, individuals already on disability pension at the time of the event and people with more than 730 consecutive sickness absence days (2years) prior to the event were excluded $(n=7547)$, resulting in a cohort of 20683 individuals. Those who died or moved abroad were excluded from the death/ emigration year onwards. This resulted in a final sample of 20498 individuals for analyses of the onset of disability pension during the first postevent year (185 individuals died or moved abroad during the first year), and 19771 for analysis of the onset of disability pension in the fifth postevent year (912 individuals died or moved abroad during the 5 follow-up years). Online supplementary figure 1 shows a flow chart of inclusion and exclusion criteria regarding each of the study questions.

\section{Measures}

An IHD event was based on hospitalisation for myocardial infarction or other IHD, excluding angina pectoris (ie, codes I21-I25 were included). A stroke event was based on hospitalisation for stroke (ICD-10 codes I60, I61, I63 and I64).

For the outcome, annual data on disability pension days were gathered. In Sweden, all individuals aged $30-64$, including people with no previous income, can be granted disability pension if their work capacity is permanently reduced owing to disease or injury. Individuals aged 19-29 can be granted temporary disability pension in cases of such reduced work capacity or in order to complete compulsory education.

The predictors of disability pension, all measured in the event year, were age, sex, education, economic inactivity, type of living area, family situation, birth country, mental disorder, cancer, diabetes and medical procedure during the event. Age was dichotomised as ' 50 years or less' and 'more than 50 years'. Education was classified as 'low' (<10years), 'intermediate' (10-12 years=high school) or 'high' ( $>12$ years=college or university). Economic activity was coded as 'economically active' (in paid work) or 'economically inactive' (not in paid work, including for example the unemployed, students and those on parental leave). Family situation was classified as 'married/cohabiting', 'not married/cohabiting without children' (ie, single) or 'not married/cohabiting with children' (ie, single parent). Birth country was dichotomised into 'Sweden' or 'country other than Sweden'. Type of living area was classified as 'large city', 'medium-sized town' or 'small town/village'.

Cancer (ICD-10 codes C00-D48) was based on information in the cancer register, and mental disorders (F00F99) and diabetes (E10-E14) were based on information from the patient register (inpatient and specialised outpatient care). All the diseases were coded 'yes' or 'no'.

Medical procedures at T-1 (year prior to the event) or T1 (year after the event) included coronary artery bypass graft, percutaneous coronary intervention, other coronary distension procedure or intravenous intracranial 
procedure. People who had undergone at least one such procedure were coded 'yes' and those without 'no'.

\section{Statistical analysis}

The cumulative incidence trend in disability pension 5 years before and 5 years after the event was calculated with frequencies (percentage of individuals on disability pension each year, with $95 \%$ CI). Between-group differences in disability pension were tested with $\chi^{2}$ tests. To assess the risk of new disability pension during the first year after the event (outcome incidence $3 \%$ ), we used generalised linear model with binary distribution and logit link function, which produced ORs with 95\% CI. To examine the differences between the predictors of disability pension for IHD and stroke cases, we tested the effect modification (interaction) of event type (IHD/ stroke) and each of the predictors. When a statistically significant $(\mathrm{p}<0.05)$ interaction effect was observed, we performed stratified subgroup analyses. The relative and absolute differences in disability pensioning by these subgroups were illustrated with least square means adjusted for all predictor variables. These adjusted means were produced using Poisson distribution due to conversion problems with binary logistic models.

In sensitivity analyses, we used generalised linear model with Poisson distribution and log link function to produce relative risks with $95 \% \mathrm{CI}$ to estimate predictors of disability pension by the fifth year after the cardiovascular event (outcome incidence 18\%). Different regression methods were used for the fifth and the first postevent years since OR is not a good approximation of risk ratio when outcome prevalence is above $10 \% .{ }^{15-17}$ SAS V.9.4 was used for all analyses.

\section{RESULTS}

\section{Cumulative incidence of disability pension}

Figure 1 illustrates the cumulative incidence of disability pension 5 years before and 5 years after a cardiovascular event of IHD or stroke: the cumulative incidence of disability pension was similar (up to $25 \%$ ) until the event for both IHD and stroke. Thus, about a quarter of

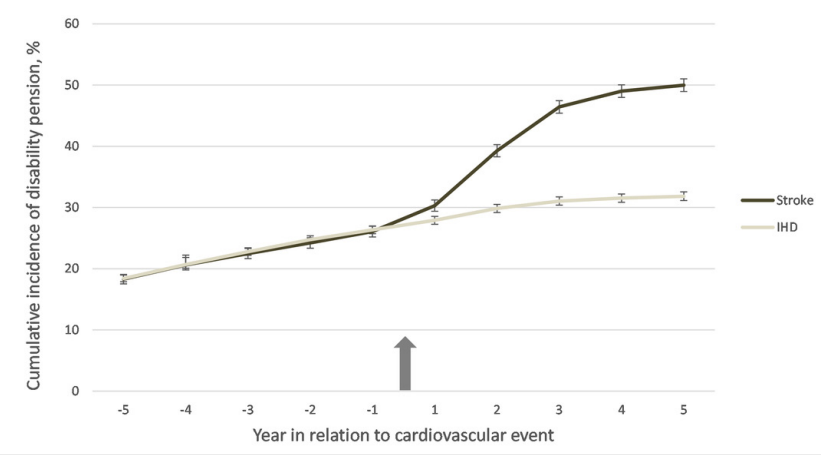

Figure 1 Cumulative incidence of disability pension 5 years before and 5 years after cardiovascular event, unadjusted. The arrow indicates the event. IHD, ischaemic heart disease. working-age people who had suffered incident IHD or a stroke were already on disability pension before the event. The highest prevalence of pre-event disability pension was observed among women $(37 \%)$, people who were economically inactive $(69 \%)$, had low education $(36 \%)$, were born outside Sweden $(35 \%)$, and had comorbid cancer $(36 \%)$, mental disorder $(58 \%)$ or diabetes $(48 \%)$ at the event year (table 1).

After the event, the cumulative incidence of disability pension was substantially higher (reaching 50\%) among people who suffered a stroke event than among those who suffered an IHD event (slightly above 30\%) (figure 1). Similar characteristics were associated with first and fifth postevent year disability pensioning, as observed before the event (table 1.)

\section{New-onset disability pension in first postevent year}

Table 2 presents both the unadjusted and adjusted results on factors associated with the risk of disability pension during the first postevent year. After adjustment for sociodemographic factors, comorbid conditions and medical procedures, patients with stroke were at a higher risk of disability pension during the first postevent year than people who had suffered an IHD event (OR=2.79; 95\% CI 2.37 to 3.29 ). Among patients with both IHD and stroke, older age $(\mathrm{OR}=1.66 ; 95 \% \mathrm{CI} 1.38$ to 1.98$)$, low education ( $\mathrm{OR}=1.58 ; 95 \% \mathrm{CI} 1.27$ to 1.97$)$, economic inactivity $(\mathrm{OR}=3.40 ; 95 \% \mathrm{CI} 2.85$ to 4.04$)$, being single without children $(\mathrm{OR}=1.25 ; 95 \% \mathrm{CI} 1.06$ to 1.48$)$, birth country other than Sweden $(\mathrm{OR}=1.27 ; 95 \% \mathrm{CI} 1.04$ to 1.55$)$, living in small towns ( $\mathrm{OR}=1.32 ; 95 \% \mathrm{CI} 1.08$ to 1.61$)$ and comorbid cancer $(\mathrm{OR}=1.85 ; 95 \%$ CI 1.27 to 2.69$)$ were associated with higher odds of disability pension in the first postevent year.

\section{Differences between IHD and stroke}

The following interactions with event type were significant: sex, mental disorder, diabetes and medical procedure. Women who had suffered an IHD event had $1.62(95 \%$ CI 1.25 to 2.11) times higher odds of disability pension in the first postevent year than male patients with IHD, whereas sex was not associated with disability pension among patients with stroke. Among IHD cases, mental disorder was associated with 3.60 (95\% CI 2.69 to 4.83) times higher odds of disability pension during the first postevent year compared with people without a mental disorder, whereas the corresponding OR among stroke cases was 1.90 (95\% CI 1.41 to 2.55$)$. Comorbid diabetes was associated with $2.49(95 \%$ CI 1.85 to 3.34$)$ times higher odds of disability pension, while it was not associated with the risk of disability pension among people who had suffered a stroke. Among stroke cases, having undergone a medical procedure was associated with $2.13(95 \%$ CI 1.33 to 3.42 ) times higher odds of disability pension in the first year after the event than among those who did not receive such procedure (table 2 ). These interactions, and absolute differences between IHD and stroke cases, are further illustrated in figure 2 , where we present 


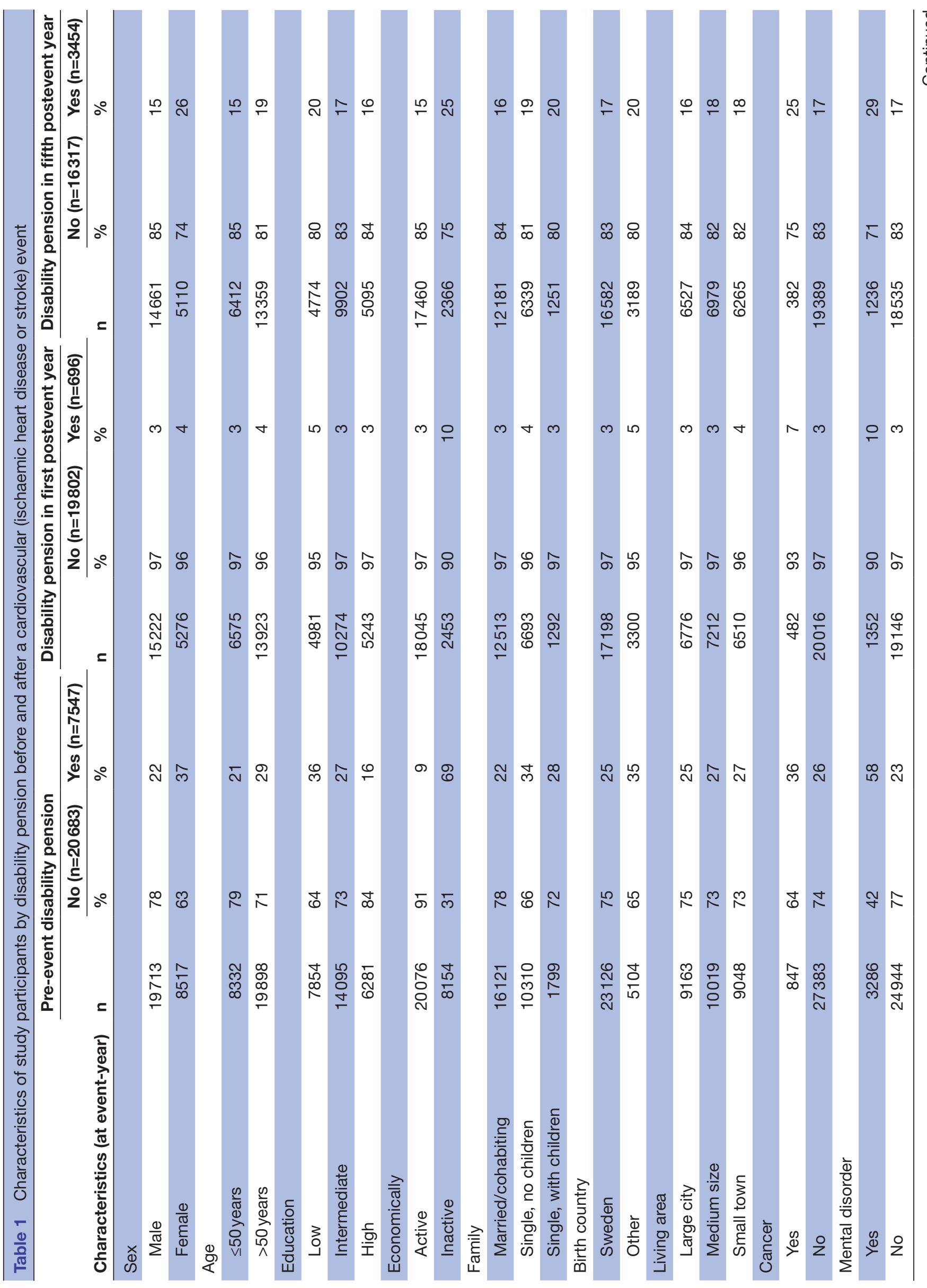




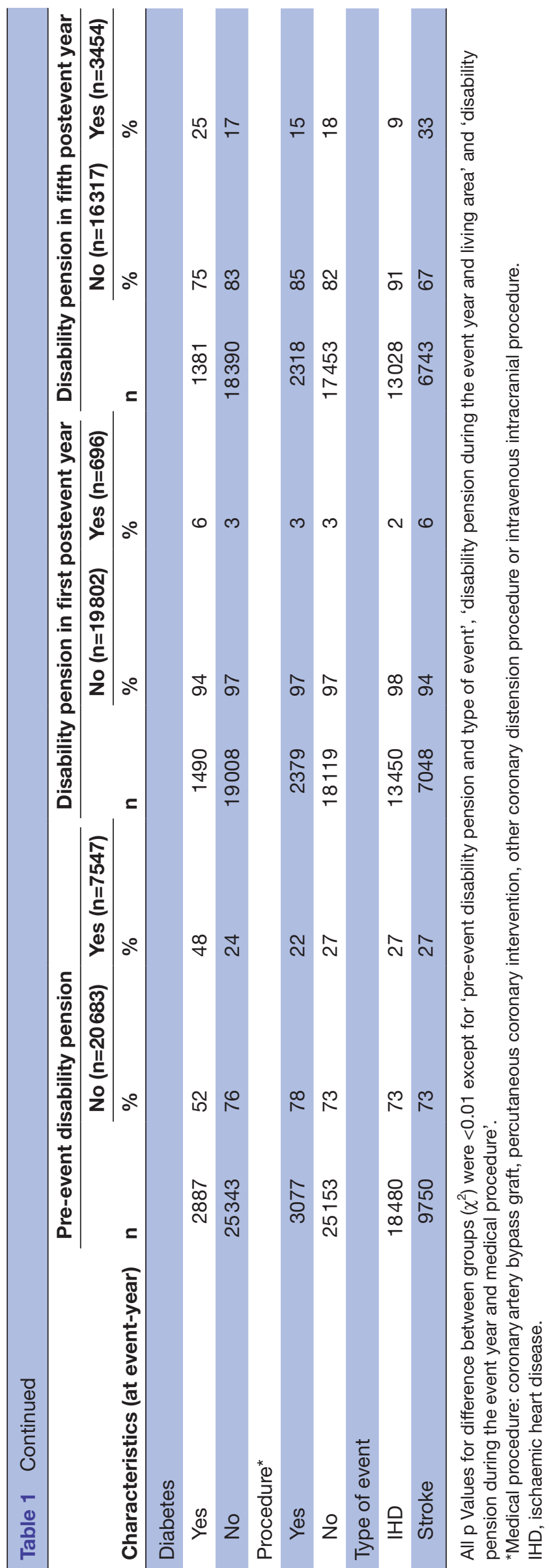

percentages of those who ended up on disability pension adjusted for other predictor variables.

Sensitivity analysis: disability pension in fifth postevent year Online supplementary table 1 presents the results regarding the factors associated with the risk of disability pension in the fifth postevent year after an IHD or stroke event. The main effects corresponded to those in first postevent year, but effect modification by event type was observed more often, indicating larger differences between IHD and stroke regarding disability pension in the fifth postyear. Interaction terms observed at first postyear remained statistically significant, but also several other interactions emerged. Those with less education, economically inactive and who were born outside of Sweden were at a higher risk of disability pension, especially among the IHD cases.

\section{DISCUSSION}

In this population-based longitudinal cohort study of people of working ages in Sweden who had a new IHD or stroke event, we found that the incidence of disability pension was similar 5 years before the first IHD or stroke event. About $25 \%$ of the cohort was already on disability pension 1 year prior to the event, with significant over-representation of socioeconomically disadvantaged. This corresponds to previous studies which have reported pre-event disability pension prevalence of $22 \%-29 \%{ }^{3}{ }^{38-20}$ We showed that similar sociodemographic characteristics and pre-existing comorbid conditions were associated with pre-event and postevent disability pension.

People who had suffered a stroke had a substantially higher incidence of disability pension after the event (up to $50 \%$ during the 5 subsequent years) than people who had suffered an IHD event (up to 30\%). Thus, although the incidence of an IHD event (18 480 cases in 3years) was more common than the incidence of stroke (9750 cases in 3 years), the disability burden of stroke was greater than that of IHD.

Female sex, older age, lower education, economic inactivity, immigrant status, living in rural areas and having comorbid conditions were all risk factors for disability pension after cardiovascular events, which corresponds to previous studies. ${ }^{4579-1221}$ The risk of disability pension after the event was higher among women than among men with IHD, but we observed no sex difference regarding stroke. Other research has reported significantly better long-term prognosis among women, ${ }^{22}$ but no sex difference in mortality due to stroke. ${ }^{23}$ Thus, the higher risk of disability pension after an IHD event among women may reflect women's higher probability of disability pension in general, ${ }^{24}$ or may be related to men's higher risk of cardiovascular mortality before disability pension is granted.

As comorbid conditions contributed to exit to disability pension, it is possible that part of these disability pensions was due to diagnoses other than cardiovascular diseases. However, as the incidence of disability pension increased 


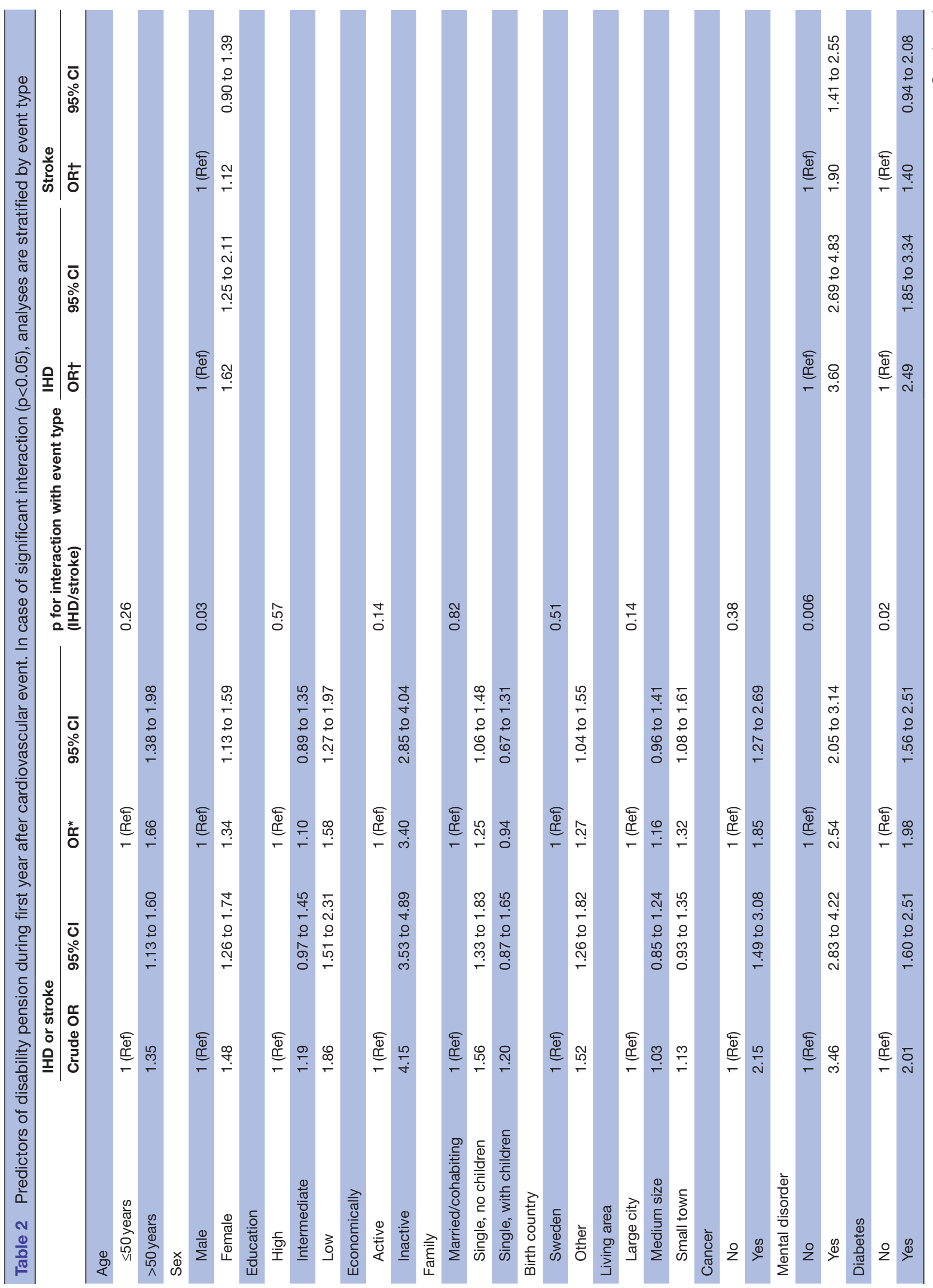



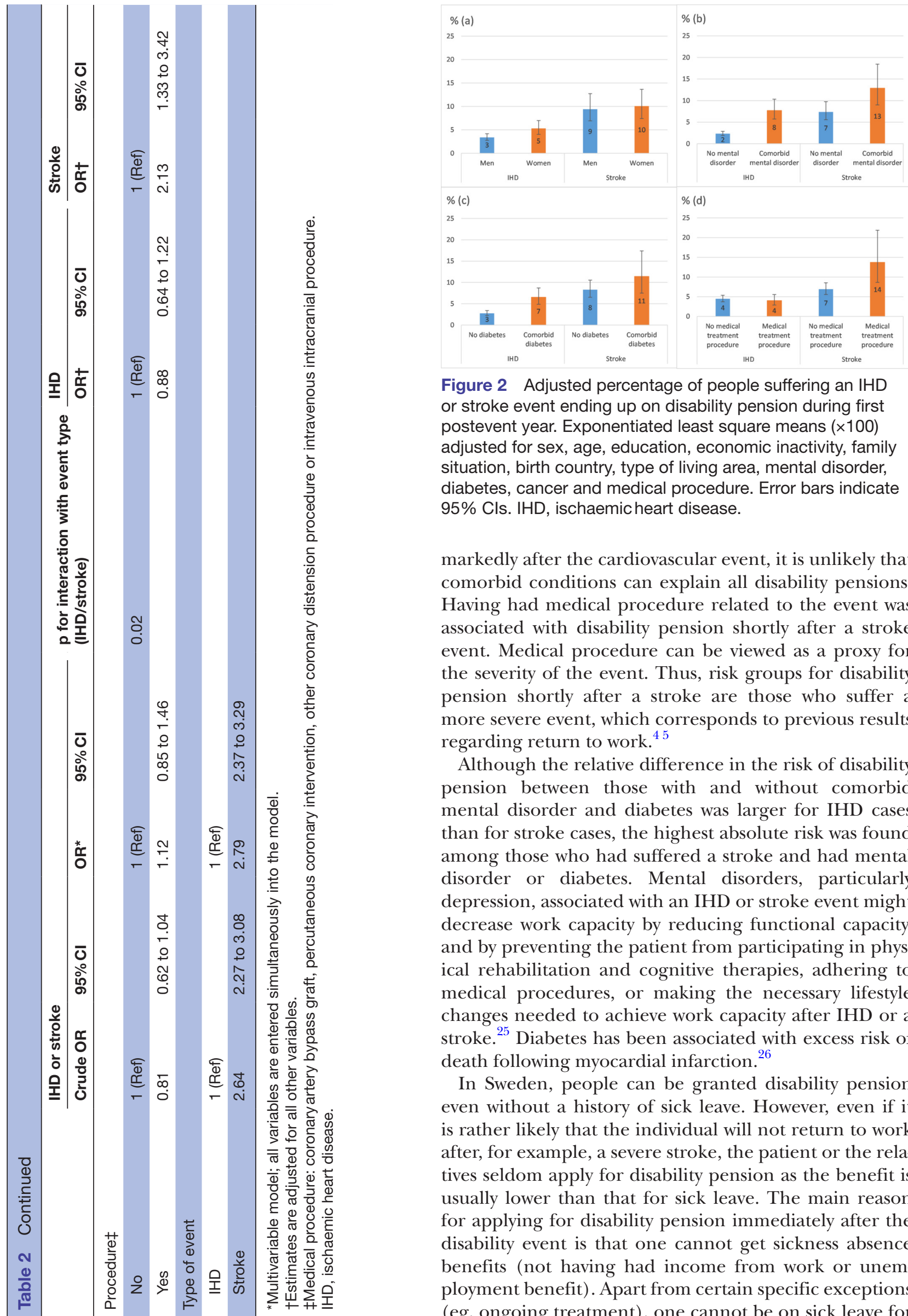

Figure 2 Adjusted percentage of people suffering an IHD or stroke event ending up on disability pension during first postevent year. Exponentiated least square means ( $\times 100)$ adjusted for sex, age, education, economic inactivity, family situation, birth country, type of living area, mental disorder, diabetes, cancer and medical procedure. Error bars indicate 95\% Cls. IHD, ischaemic heart disease.

markedly after the cardiovascular event, it is unlikely that comorbid conditions can explain all disability pensions. Having had medical procedure related to the event was associated with disability pension shortly after a stroke event. Medical procedure can be viewed as a proxy for the severity of the event. Thus, risk groups for disability pension shortly after a stroke are those who suffer a more severe event, which corresponds to previous results regarding return to work. ${ }^{45}$

Although the relative difference in the risk of disability pension between those with and without comorbid mental disorder and diabetes was larger for IHD cases than for stroke cases, the highest absolute risk was found among those who had suffered a stroke and had mental disorder or diabetes. Mental disorders, particularly depression, associated with an IHD or stroke event might decrease work capacity by reducing functional capacity, and by preventing the patient from participating in physical rehabilitation and cognitive therapies, adhering to medical procedures, or making the necessary lifestyle changes needed to achieve work capacity after IHD or a stroke. ${ }^{25}$ Diabetes has been associated with excess risk of death following myocardial infarction. ${ }^{26}$

In Sweden, people can be granted disability pension even without a history of sick leave. However, even if it is rather likely that the individual will not return to work after, for example, a severe stroke, the patient or the relatives seldom apply for disability pension as the benefit is usually lower than that for sick leave. The main reason for applying for disability pension immediately after the disability event is that one cannot get sickness absence benefits (not having had income from work or unemployment benefit). Apart from certain specific exceptions (eg, ongoing treatment), one cannot be on sick leave for 
more than 365 consecutive days. Thus, people who were granted disability pension during the first postevent year were possibly in a poorer labour market position, which prevented them from applying for sickness absence benefits. This corresponds to our findings, since economic inactivity was the strongest predictor of disability pension in the first postevent year regardless of event type. Other indicators of poorer labour market position, such as low education and birth country other than Sweden, were also predictive of fast exit to disability pension.

Socioeconomic background and comorbid conditions explained the risk of disability pension 5 years after the event to a greater extent among IHD than stroke cases. This is noteworthy, since poorer labour market position and not fulfilling the criteria for entitlement to sickness absence benefits cannot explain disability pension in the fifth postevent year. The often higher severity of stroke compared with IHD may explain this difference; after an IHD event, the probability of recovering to relatively good work capacity may be higher. However, the observed differences in this recovery seem to relate to socioeconomic characteristics and resources; the background factors may affect people's recovery and rehabilitation. ${ }^{27}$ Stroke, often a more disabling cardiovascular event, may more totally reduce work capacity, and hence we found smaller individual differences. However, a socioeconomic gradient has also been observed in short and long-term outcomes after a stroke. ${ }^{28}$

The major strength of this study was its large population-based cohort data with reliable register-based measures of high coverage and specificity, ${ }^{29}$ and no loss to follow-up. Compared with previous studies, we also had a longer follow-up- 5 years-both before and after the event. We were able to include a large set of predictors of disability pension, including sociodemographic factors, comorbid conditions and medical procedure.

The register data also have some limitations: we were only able to include information that was available in administrative registers. This meant that we had no information on quality and outcome of postevent care, individuals' health behaviours or workplace psychosocial factors, which are typically collected in surveys, and have previously been linked to disability pension in general populations. ${ }^{30}$ However, a recent study among Finnish public sector employees demonstrated that the contribution of health behaviours and workplace psychosocial factors to the risk of disability pension was relatively small compared with the contribution of comorbidity, especially mental comorbidity. ${ }^{7}$ Regarding postevent care, men were more likely to enrol in disease management programme than women after coronary heart disease in Germany. ${ }^{31}$ We also did not have direct measure of event severity, but used medical procedure as a proxy measure. In future studies, also recurrent events could be included. Finally, the high employment frequency in higher ages and among women in Sweden as well as the universal coverage with relatively high benefit levels might limit the generalisability of the results. $^{32}$
In a recent study, disability pensioning 5 years after percutaneous coronary intervention or coronary artery bypass crafting was fairly common $(15 \%-35 \%)$ among young ( $\leq 50$ years) patients with IHD. ${ }^{33}$ The fact that even after successful surgery and complete revascularisation, these patients often ended up on disability pension led the authors to speculate that disability pensioning may be partly explained by patients and healthcare professionals' attitudes towards recovery and return to work. ${ }^{33}$ In Sweden, at least one physician and often other health professionals are involved in the assessments of the disease the patient has, the functional limitations the disease has led to and to what extent those limitations actually might influence the work capacity of the patient and for how long. These assessments are sent to the Social Insurance Agency, where an officer evaluates and decides whether the patient (claimant) fulfils the criteria for being granted disability pension or not, and if so, to what extent (parttime or full-time). However, other types of studies are warranted to shed light on these processes, and perhaps this explorative study can inspire such studies.

\section{CONCLUSIONS}

Our results quantify and emphasise the burden of IHD and stroke to the labour market, and can help occupational and other healthcare professionals to identify vulnerable groups at risk for permanent exclusion from labour market after such an event. While IHD event was more common, stroke caused more permanent work disability. As regards IHD, non-medical risk factors contributed to the risk of disability pension, whereas medical factors contributed to the risk of disability pension after stroke. This knowledge may be beneficial when planning interventions to prevent permanent work disability after either event.

Contributors JE, MV, TL, EMR and KA contributed to conception and design. $\mathrm{JE}$ analysed the data and drafted the manuscript. All authors contributed either to analysis, interpretation or acquisition of the data, and critically revised the manuscript. All authors gave final approval and agree to be accountable for all aspects ensuring integrity and accuracy.

Funding This study was supported by the Swedish Research Council for Health, Working Life and Welfare. JE, MV and TL were supported by the Academy of Finland (projects 258598, 292824, 287488). The funding organisations had no role in the study design, data collection, analysis, interpretation of the data, writing the report, or in the decision to submit the paper.

Competing interests None declared.

Patient consent Obtained.

Ethics approval Ethical approval was obtained from the Regional Ethical Review Board, Stockholm, Sweden.

Provenance and peer review Not commissioned; externally peer reviewed.

Data sharing statement No additional data available.

Open Access This is an Open Access article distributed in accordance with the Creative Commons Attribution Non Commercial (CC BY-NC 4.0) license, which permits others to distribute, remix, adapt, build upon this work non-commercially, and license their derivative works on different terms, provided the original work is properly cited and the use is non-commercial. See: http://creativecommons.org/ licenses/by-nc/4.0/ 
(c) Article author(s) (or their employer(s) unless otherwise stated in the text of the article) 2017. All rights reserved. No commercial use is permitted unless otherwise expressly granted.

\section{REFERENCES}

1. GBD Compare Data Visualization. Seattle, WA: Institute for Health Metrics and Evaluation (IHME), University of Washington, 2016.

2. Schmidt M, Jacobsen JB, Lash TL, et al. 25 year trends in first time hospitalisation for acute myocardial infarction, subsequent short and long term mortality, and the prognostic impact of sex and comorbidity: a Danish nationwide cohort study. BMJ 2012;344:e356.

3. Hämäläinen $\mathrm{H}$, Mäki J, Virta L, et al. Return to work after first myocardial infarction in 1991-1996 in Finland. Eur J Public Health 2004;14:350-3.

4. Peters GO, Buni SG, Oyeyemi AY, et al. Determinants of return to work among Nigerian stroke survivors. Disabil Rehabil 2013;35:455-9.

5. Bonner B, Pillai R, Sarma PS, et al. Factors predictive of return to work after stroke in patients with mild-moderate disability in India. Eur J Neurol 2016;23:548-53.

6. Catalina-Romero C, Ruilope LM, Sánchez-Chaparro MA, et al. Factors influencing return-to-work after cerebrovascular disease: the importance of previous cardiovascular risk. Eur J Prev Cardiol 2015;22:1220-7

7. Ervasti J, Kivimäki M, Pentti J, et al. Health- and workrelated predictors of work disability among employees with a cardiometabolic disease-A cohort study. J Psychosom Res 2016;82:41-7.

8. Lundbom J, Myhre HO, Ystgaard B, et al. Factors influencing return to work after aortocoronary bypass surgery. Scand $J$ Thorac Cardiovasc Surg 1992;26:187-92.

9. Jespersen L, Abildstrøm SZ, Hvelplund A, et al. Symptoms of angina pectoris increase the probability of disability pension and premature exit from the workforce even in the absence of obstructive coronary artery disease. Eur Heart J 2013;34:3294-303.

10. Osler M, Mårtensson S, Prescott E, et al. Impact of gender, comorbidity and social factors on labour market affiliation after first admission for acute coronary syndrome. A cohort study of Danish patients 2001-2009. PLoS One 2014;9:e86758.

11. Zetterström K, Vaez M, Alexanderson K, et al. Disability pension after coronary revascularization: a prospective nationwide register-based Swedish cohort study. Eur J Prev Cardiol 2015;22:304-11.

12. Gunn J, Kiviniemi T, Biancari F, et al. Predictors of permanent work disability among $\leq 50$-year-old patients undergoing percutaneous coronary intervention. Scand J Work Environ Health 2015;41:460-6.

13. Hamer M, Batty GD, Stamatakis E, et al. Comparison of risk factors for fatal stroke and ischemic heart disease: a prospective follow up of the health survey for England. Atherosclerosis 2011;219:807-10.

14. International Statistical Classification of Diseases and Related Health Problems (ICD-10). Geneva, Switzerland: World Health Organization 1994.

15. Deddens JA, Petersen MR. Approaches for estimating prevalence ratios. Occup Environ Med 2008:65:501-6.
16. Greenland S. Model-based estimation of relative risks and other epidemiologic measures in studies of common outcomes and in case-control studies. Am J Epidemiol 2004;160:301-5.

17. Zou G. A modified poisson regression approach to prospective studies with binary data. Am J Epidemiol 2004;159:702-6.

18. Teasdale TW, Engberg AW. Disability pensions in relation to stroke: a population study. Brain Inj 2002;16:997-1009.

19. Medin J, Nordlund A, Ekberg K. Sick leave, disability pension and health-care-seeking behaviour prior to stroke, among people aged 30-65: a case-control study. Brain Inj 2007;21:457-63.

20. Zetterström K, Voss M, Alexanderson K, et al. Disability Pension at the Time of Coronary Revascularisation Is Associated with Higher Five-Year Mortality; A Swedish Nationwide, Register-Based Prospective Cohort Study. PLoS One 2015;10:e0135277.

21. Dreyer RP, Xu X, Zhang W, et al. Return to Work After Acute Myocardial Infarction: Comparison Between Young Women and Men. Circ Cardiovasc Qual Outcomes 2016;9:S45-52.

22. van der Meer MG, Cramer MJ, van der Graaf Y, et al. Gender difference in long-term prognosis among patients with cardiovascular disease. Eur J Prev Cardiol 2014;21:81-9.

23. Mosca L, Barrett-Connor E, Wenger NK. Sex/gender differences in cardiovascular disease prevention: what a difference a decade makes. Circulation 2011;124:2145-54.

24. Falkstedt D, Backhans M, Lundin A, et al. Do working conditions explain the increased risks of disability pension among men and women with low education? A follow-up of Swedish cohorts. Scand $J$ Work Environ Health 2014;40:483-92.

25. Kutlubaev MA, Hackett ML. Part II: predictors of depression after stroke and impact of depression on stroke outcome: an updated systematic review of observational studies. Int J Stroke 2014;9:1026-36.

26. Alabas OA, Hall M, Dondo TB, et al. Long-term excess mortality associated with diabetes following acute myocardial infarction: a population-based cohort study. J Epidemiol Community Health 2017;71:25-32.

27. Myers V, Drory Y, Goldbourt U, et al. Multilevel socioeconomic status and incidence of frailty post myocardial infarction. Int $J$ Cardiol 2014;170:338-43.

28. Marshall IJ, Wang Y, Crichton S, et al. The effects of socioeconomic status on stroke risk and outcomes. Lancet Neurol 2015;14:1206-18.

29. Ludvigsson JF, Andersson E, Ekbom A, et al. External review and validation of the Swedish national inpatient register. BMC Public Health 2011;11:450.

30. Albertsen K, Lund T, Christensen KB, et al. Predictors of disability pension over a 10-year period for men and women. Scand J Public Health 2007;35:78-85.

31. Bozorgmehr K, Maier W, Brenner $\mathrm{H}$, et al. Social disparities in Disease Management Programmes for coronary heart disease in Germany: a cross-classified multilevel analysis. J Epidemiol Community Health 2015;69:1091-101.

32. Arts W, Gelissen J. Three worlds of welfare capitalism or more? A state-of-the-art report. Journal of European Social Policy 2002;12:137-58.

33. Lautamäki A, Gunn JM, Airaksinen KEJ, et al. Permanent work disability in patients $\leq 50$ years old after percutaneous coronary intervention and coronary artery bypass grafting (the CRAGS study). Eur Heart J Qual Care Clin Outcomes 2017;3:101-6. 